Annales Academiæ Scientiarum Fennicæ

Series A. I. Mathematica

Volumen 14, 1989, 291-313

\title{
UNCOUNTABLE-ORDER SETS FOR RADIAL-LIMIT FUNCTIONS
}

\section{Robert Berman, Togo Nishiura, and George Piranian}

\section{Introduction and synopsis}

Let $\Delta$ and $C$ denote the unit disk $\{|z|<1\}$ and the unit circle $\{|z|=1\}$. Corresponding to each analytic function $f$ on $\Delta$, let $f^{*}$ denote the radial-limit function associated with $f$; that is, let $f^{*}(\zeta)=\lim _{r \rightarrow 1} f(r \zeta)$ for each point $\zeta$ on $C$ for which the limit exists as a finite or infinite value.

Definition. If $g$ is a mapping of some set into a space $Y$, we call a point $y$ in $Y$ an uncountable-order value for $g$ provided the set $g^{-1}(y)$ is uncountable; we call the set of uncountable-order values for $g$ the uncountable-order set for $g$, and we denote it by $U(g)$.

In [12], G.R. MacLane and F.B. Ryan exhibited a Blaschke product $B$ such that the set $U\left(B^{*}\right)$ coincides with an arbitrarily prescribed closed set on $C$. By constructing appropriate Riemann surfaces over $\Delta$, they proved the following proposition.

Theorem A. Corresponding to each closed set $W$ on the unit circle there exists a Blaschke product $B$ such that $U\left(B^{*}\right)=W$ and $B^{*}(\zeta)$ has modulus 1 wherever it exists.

MacLane and Ryan asked whether it is feasible to prove Theorem A by describing suitable Blaschke products in terms of their zeros. We answer this question in the affirmative. Also, we characterize the sets on $C$ that serve as the set $U\left(f^{*}\right)$ for some inner function $f$, in other words, for some bounded analytic function $f$ with $\left|f^{*}(\zeta)\right|=1$ almost everywhere on $C$ (see Theorem 3). For the sake of clarity, we begin with an analogous problem in a larger class of functions.

Theorem 1. A set $W$ in the extended plane $\hat{\mathbf{C}}$ is the set $U\left(f^{*}\right)$ for some (bounded) analytic function $f$ on $\Delta$ if and only if it is a (bounded) analytic set.

(The class of analytic sets will be defined in Section 2.)

Our Theorems 1 and 3 resemble results published by Ryan in two papers ([19] and [20]) devoted to the characterization of sets that serve as the sets of asymptotic values of analytic functions of various classes.

In Section 2, we deal with topological aspects of Theorem 1. We prove the necessity of the condition in the theorem, and we show, by modifying a result of 
S. Mazurkiewicz and W. Sierpiński [13], that to each nowhere-dense perfect set $P$ on $C$ there corresponds a continuous function $g$ on $P$ (with appropriate range) such that $U(g)=W$.

In Section 3, we complete the proof of the sufficiency of the condition in Theorem 1 (see the proof of Theorem $1^{\prime}$ ); we choose a thin perfect set $P$ on $C$ and show that some analytic function $f$ on $\Delta$ (bounded if $W$ is bounded) has an analytic extension across each arc of $C \backslash P$ and agrees with $g$ on $P$. If $W$ is bounded, the existence of the required function $f$ follows from a theorem of W. Rudin [18]. In the case where $W$ is unbounded, we invoke Lemma 7; this is an analogue of Rudin's theorem for general analytic functions; it is based on an approximation theorem of N.U. Arakeljan [1]. In either case, the verification that $U\left(f^{*}\right)=U(g)=W$ is easy. Using Lemma 7, we also prove the following result, which overlaps with a theorem of F. Herzog and G. Piranian [7].

Theorem 2. To each nowhere dense set $H$ of type $G_{\delta \sigma}$ on $C$ there corresponds an analytic function $f$ on $\Delta$ such that $f^{*}$ exists everywhere on $C \backslash H$ and nowhere on $H$.

The problem of MacLane and Ryan calls for geometric estimates on Blaschke products whose zeros approach the unit circle rapidly. In Section 4, we establish Lemmas 9 and 10. The first of these deals with the behavior at a fixed point $\zeta$ on $C$ of a single Blaschke factor as its zero moves along a circular arc in $\Delta$ that separates the points 0 and $\zeta$. The second gives an upper bound for the quantity $|1-B(z)|$ for a Blaschke product $B$ whose zeros lie much nearer to $C$ than to the point $z$. In Section 5, we use Lemmas 9 and 10 to prove Lemma 11, an analogue of Rudin's theorem for inner functions. By means of Lemma 11, we then prove the following extension of Theorem A.

Theorem 3. There exists a Blaschke product $\tilde{B}$ such that for each analytic set $W$ on $C$ some subproduct $B$ of $\tilde{B}$ satisfies the conditions (i) $U\left(B^{*}\right)=W$ and (ii) $\left|B^{*}(\zeta)\right|=1$ for each point $\zeta$ on $C$.

Lemma 11 also serves in the proof of the following result, which contains an analogue for Blaschke products of Theorem 2.

Theorem 4. To each uncountable analytic set $E$ on $C$ correspond a nonempty perfect subset $P$ of $E$ and a Blaschke product $\tilde{B}$, analytic on $C \backslash P$, such that if $H$ is a $G_{\delta \sigma}$-set in $P$, then some subproduct of $\tilde{B}$ has a radial limit at each point of $C \backslash H$ and at no point of $H$.

Finally, Section 6 treats two variants of our central problem. If $g: X \rightarrow Y$ denotes a function defined in a measure space $X$ (or in a topological space $X$ ), we call a point $y$ in $Y$ a positive-measure value for $\mathrm{g}$ (or a second-category value for $g$ ) provided the set $g^{-1}(y)$ has positive measure (or is of second category). A theorem of F. Riesz and M. Riesz [16] asserts that the set of positive-measure values for the 
radial-limit function associated with a nonconstant bounded analytic function is empty, and a theorem of G.T. Cargo [2, Theorem 1] aserts that the set of secondcategory values for the radial-limit function associated with a nonconstant inner function is also empty. The additivity of Lebesgue measure implies that even in the more general space of Borel-measurable complex-valued functions mapping a Borel subset of the circle $C$ into $\hat{\mathbf{C}}$, each function can have at most countably many positive-measure values. Similarly, because every family of disjoint Borel sets of second category on $C$ is at most countable (see Lemma 5 in Section 2), each function can have at most countably many second-category values. The following two theorems are therefore sharp.

Theorem 5. Every countable set in $\hat{\mathbf{C}}$ is the set of positive-measure values for the radial-limit function $f^{*}$ associated with some analytic function $f$ on $\Delta$.

Theorem 6. Every (bounded) countable set in $\hat{\mathbf{C}}$ is the set of secondcategory values for the radial-limit function $f^{*}$ associated with some (bounded) analytic function $f$ on $\Delta$.

The authors are grateful to Peter Colwell, whose survey of the literature on Blaschke products [4] brought several relevant papers to their attention. Piranian also thanks the Mathematics Department of the University of Maryland; its generous hospitality during fall term of 1983 provided an environment favorable to the generation of Blaschke products.

\section{Topological considerations}

In this section we present five lemmas that will be used later in the paper. Lemmas 1 and 2 are found in references [5] and [9]. The first deals with radiallimit functions, and the second asserts the necessity of the condition in Theorem 1. Lemma 3 states a classical result of $M$. Souslin concerning analytic sets. Lemma 4 generalizes to analytic sets in $\hat{\mathbf{C}}$ and to complex-valued functions a result by Mazurkiewicz and Sierpiński [13]; in Section 3 we shall use it to complete the proof of Theorem 1 and to prove Theorem 3. Lemma 5 concerns sets of second category. Finally, we give a modified version of a theorem of $\mathrm{H}$. Hahn concerning the pointwise limit of a sequence of continuous functions.

Let us begin with a topological space $X$. We shall denote the closure of a subset $W$ of $X$ by $\bar{W}$. For the sake of completeness, we present some basic terminology and background information found in [9, Sections 36-39]. The class of Borel sets in $X$ is the smallest family of sets in $X$ that contains all closed sets and is closed under the operations of forming complements and countable intersections. Each Borel set has an order. Specifically, the open sets and the closed sets are Borel sets of order 0 , sets of type $F_{\sigma}$ and $G_{\delta}$ are Borel sets of order 1 , and so forth for all countable ordinal numbers [9, pp. 48, 344, 345]. Borel-measurable mappings also have orders. That is, if $X$ and $Y$ are topological spaces and $E$ 
is a Borel set in $X$, then a mapping $g: E \rightarrow Y$ is Borel-measurable of class $\alpha$ provided for each closed set $K$ in $Y$ the inverse image $g^{-1}(K)$ is a Borel set of order $\alpha[9$, pp. 345, 373]. It is well known that the limit of a convergent sequence of functions of class $\alpha$ is of class $\alpha+1[9, \mathrm{p} .386]$ and the limit of a convergent sequence of Borel-measurable functions is Borel-measurable.

Here we pause to state our first lemma.

Lemma 1. If $f$ is a meromorphic function on $\Delta$, then the points on $C$ where $f^{*}$ exists constitute an $F_{\sigma \delta}$-set, and $f^{*}$ is a Borel-measurable function.

For a proof of the first assertion, see [5, Section 44]; the second assertion follows from the penultimate paragraph before the lemma.

Let us now go to analytic sets.

Definition. The class of analytic sets (or Souslin sets) in a topological space $X$ is the family of images of Borel sets under continuous mappings of $X$ into $X$ $[9$, p. 453].

We shall denote by $\mathcal{N}$ the space of irrational numbers in the interval $I=[0,1]$. Because every Borel set in a complete separable metric space is a continuous image of $\mathcal{N}$ [9, Section 37, I, Theorem 1, p. 446], every analytic set in the extended plane $\hat{\mathbf{C}}$ is the image of $\mathcal{N}$ under a continuous mapping. The italicized portion of the preceding sentence will play a role in the second step of the proof of Lemma 4.

Lemma 2 (see [9, p. 496, Theorem 2]). Let $X$ and $Y$ be complete separable metric spaces, and let $g: E \rightarrow Y$ be a Borel-measurable function defined on a Borel set $E$ in $X$. Then the uncountable-order set $U(g)$ is an analytic set in $Y$.

Lemmas 1 and 2 imply immediately that if $f$ is a meromorphic function in $\Delta$, then $U\left(f^{*}\right)$ is an analytic set in $\hat{\mathbf{C}}$. The necessity of the condition in Theorem 1 follows as a special case. 479]).

The following lemma was proved by M. Souslin (see [11, p. 94] or [9, pp. 445,

Lemma 3. Every uncountable analytic set in a complete separable metric space contains a homeomorphic image of the Cantor set.

If $f$ is a meromorphic function on $\Delta$ and $w$ is a point in $\hat{\mathbf{C}}$, then the points on $C$ where $f$ has the radial limit $w$ constitute a set of type $F_{\sigma \delta}$. Together with Lemma 3 , this implies that each uncountable-order value for $f^{*}$ has $2^{\aleph_{0}}$ preimages.

Mazurkiewicz and Sierpiński [13] showed that every analytic set on the real line $\mathbf{R}$ is the uncountable-order set for some continuous function $g: \mathbf{R} \rightarrow \mathbf{R}$. By means of a slight modification in their proof, we shall now obtain the following result. 
Lemma 4. Let $P$ be a nonempty, perfect, nowhere-dense set on $C$, and let $W$ be an analytic set in $\hat{\mathbf{C}}$. If $W \neq \emptyset$, then there exists a continuous function $g: P \rightarrow W$ such that $U(g)=W$. If $W=\emptyset$, then corresponding to each uncountable analytic set $S$ in $\hat{\mathbf{C}}$ there exists a continuous function $g: P \rightarrow S$ such that $U(g)=\emptyset=W$.

In the special case where $W=\emptyset$, let $S$ denote an uncountable analytic set in $\hat{\mathbf{C}}$. By Lemma 3, there exists a homeomorphism $g$ of $P$ onto a perfect subset of $S$. Because $g$ takes no value more than once, $U(g)=\emptyset=W$.

For the case where $W \neq \emptyset$, we shall divide the proof into four steps. In the first step, we construct two continuous mappings $\mu$ and $h$. They are independent of each other and of the sets $P$ and $W$, and they will remain fixed throughout the remainder of the proof.

Let $\lambda$ denote the standard continuous mapping of the Cantor set $K$ onto the interval $I$ (see [17, pp. 47-48]). Since the mapping $\lambda$ is at most 2 -to- 1 , the continuous mapping $\mu: K^{4} \rightarrow I^{4}$ defined by the formula

$$
\mu(p, q, r, s)=(\lambda(p), \lambda(q), \lambda(r), \lambda(s))
$$

is obviously at most 16 -to- 1 .

The radial projection of a cubical surface onto the Riemann sphere leads in a natural way to a homeomorphism $h$ of the set $I \times \partial I^{3}$ in $I^{4}$ onto $I \times \hat{\mathbf{C}}$.

The second step is the construction of a continuous mapping $\varphi: \mathcal{N} \rightarrow \hat{\mathbf{C}}$ such that $U(\varphi)=W$. By a theorem of N.N. Lusin (see [13, p. 162] or [9, Remark 1 on p. 497]), there exists a continuous mapping $\gamma: \mathcal{N} \rightarrow \mathcal{N}$ such that $U(\gamma)=\mathcal{N}$. Also, by the italicized assertion following the definition of analytic sets, there exists a continuous mapping $\beta: \mathcal{N} \rightarrow W$. The composite mapping $\varphi=\beta \circ \gamma$ of $\mathcal{N}$ onto $W$ has the required properties.

The third step begins with the graph $G$ of the function $\varphi$ constructed in the second step. Clearly,

$$
G=\{(t, f(t)): t \in \mathcal{N}\} \subset \mathcal{N} \times \hat{\mathbf{C}} \subset I \times \hat{\mathbf{C}} .
$$

Let $\bar{G}$ denote the closure of $G$ in $I \times \hat{\mathbf{C}}$, let $\pi: I \times \hat{\mathbf{C}} \rightarrow \hat{\mathbf{C}}$ denote the usual projection mapping, and let $\psi: \bar{G} \rightarrow \hat{\mathbf{C}}$ denote the restriction of $\pi$ to $\bar{G}$. Because $\varphi$ is continuous, $G$ is closed relative to $\mathcal{N} \times \hat{\mathbf{C}}$. Therefore the countability of $I \backslash \mathcal{N}$ implies that $U(\psi)=U(\varphi)$.

We begin the fourth step with the observation that because the set $\bar{G}$ in $I \times \hat{\mathbf{C}}$ is closed and uncountable, the inverse image $h^{-1}(\bar{G})$ in $I^{4}$ is also closed and uncountable. From this it follows that the set $\mu^{-1}\left[h^{-1}(\bar{G})\right]$ in $K^{4}$ is closed and uncountable; let $Q$ denote its perfect part. Because $Q$ is not empty, some homeomorphism $\nu$ maps the perfect set $P$ of Lemma 4 onto $Q$. Clearly, the set 
$h \circ \mu \circ \nu(P)$ lies in $\bar{G}$. Using the function $\psi$ defined in the third step, we now define the mapping

$$
g=\psi \circ h \circ \mu \circ \nu: P \rightarrow \hat{\mathbf{C}} .
$$

Since the set $\nu(P)=Q$ contains all except at most countably many points of $\mu^{-1}\left[h^{-1}(G)\right]$, it is clear that $U(g)=U(\psi)=U(\varphi)=W$.

This concludes the proof of Lemma 4. The line

$$
P \stackrel{\nu}{\longrightarrow} \mu^{-1}\left[h^{-1}(\bar{G})\right] \subset K^{4} \stackrel{\mu}{\longrightarrow} I^{4} \stackrel{h}{\longrightarrow} I \times \hat{\mathbf{C}} \supset \bar{G} \stackrel{\psi}{\longrightarrow} \hat{\mathbf{C}}
$$

provides a summary of the four steps.

Next we shall discuss sets of second category.

Lemma 5. Every family of disjoint Borel sets of second category in a space with a countable basis consists of at most countably many elements.

Proof. By a theorem of H. Lebesgue ([10, p. 187], [9, Corollary on p. 88]), every Borel set $E$ in a space $X$ is congruent to some open set $G$, modulo the sets of first category (in other words, $E=(G \backslash V) \cup W$, where $V$ and $W$ are sets of first category). Because the interior of a set of first category is empty, two disjoint Borel sets are congruent to disjoint open sets. It follows that in a space with a countable basis every family of disjoint Borel sets has at most countably many elements of second category.

Suppose now that $E$ is a Borel set in a space $X$ with a countable basis and that $g: E \rightarrow Y$ is a Borel-measurable function. Then there exist at most countably many points $y$ in $Y$ for which $g^{-1}(y)$ is of second category in $X$. In particular, it follows that the set of second-category values of the radial-limit function $f^{*}$ of an analytic function $f$ in $\Delta$ is at most countably infinite (see Theorem 6).

To close this section, we state without proof a slightly modified version of a theorem of H. Hahn (see [5, Section 44, Theorem I]): Corresponding to each set of type $F_{\sigma \delta}$ on $C$, there exists a sequence of continuous mappings $g_{n}: C \rightarrow C$ such that $\lim _{n \rightarrow \infty} g_{n}(\zeta)$ exists if and only if $\zeta \in E$.

\section{Application of an approximation theorem of Arakeljan and a theorem of Rudin}

In this section we establish the sufficiency of the condition in Theorem 1. For completeness, we shall state Arakeljan's theorem. In the following formulation, we denote Alexandrov's one-point compactification $G \cup\left\{z_{\infty}\right\}$ of a domain $G$ in $\hat{\mathbf{C}}$ by $G^{\infty}$.

Lemma 6 (Arakeljan [1]). Let $G$ be a domain in $\hat{\mathbf{C}}$, let $K$ be a relatively closed, nowhere-dense set in $G$ such that the set $G^{\infty} \backslash K$ is connected and locally connected, and let $h$ denote a continuous, complex-valued function on $K$. Then, corresponding to each positive continuous function $\varepsilon$ on $K$, there exists an analytic function $f$ on $G$ such that $|f(z)-h(z)|<\varepsilon(z)$ for all $z$ in $K$. 
The next lemma will enable us to progress from the construction of the continuous function $g$ in Lemma 4 to the construction of an analytic function having the same uncountable-order set as $g$. It will also allow us to prove Theorem 2 . We denote by $\sigma$ the spherical metric on $\hat{\mathbf{C}}$, that is, the metric on $\hat{\mathbf{C}}$ induced by the stereographic projection of $\hat{\mathbf{C}}$ onto the Riemann sphere.

Lemma 7. Let $N$ be a nonempty, closed, nowhere-dense set on the circle $C$, and let $\left\{g_{n}\right\}$ be a sequence of continuous functions mapping $C$ into $\hat{\mathbf{C}}$. Then there exists a nonconstant function $f$ such that

(i) $f$ is analytic on $\bar{\Delta} \backslash N$, and

(ii) at each point $\zeta$ of $N$ the radial cluster set of $f$ at $\zeta$ contains the set of cluster points of the sequence $\left\{g_{n}(\zeta)\right\}$ and is equal to it if

$$
\lim _{n \rightarrow \infty} \sigma\left[g_{n}(\zeta), g_{n+1}(\zeta)\right]=0 .
$$

Clearly, at each point $\zeta$ on $C$, the function $f$ described in the lemma has a radial limit if and only if $\zeta \in C \backslash N$ or $\lim _{n \rightarrow \infty} g_{n}(\zeta)$ exists.

We start by making several reductions on the sequence $\left\{g_{n}\right\}$.

First, since we are concerned only with its behavior on $N$, we can suppose that the functions $g_{n}$ are defined (and continuous) only on $N$.

Without loss of generality, we may assume that the function $g_{1}$ is constant and finite. Moreover, we can choose a sequence $\left\{\tilde{g}_{n}\right\}$ of functions on $N$ satisfying the following four conditions. (i) The function $\tilde{g}_{1}$ is identical with $g_{1}$, and each of the functions $\tilde{g}_{n}$ is continuous and assumes only finitely many different values, all finite. (ii) For some point $\zeta$ in $N$, the spherical distance $\sigma\left(\tilde{g}_{1}(\zeta), \tilde{g}_{2}(\zeta)\right)$ has a positive value $\sigma_{0}$. (iii) For each point $\zeta$ on $N$ and each index $n$, every great circle through the stereographic images of the two points $\tilde{g}_{n}(\zeta)$ and $\tilde{g}_{n+1}(\zeta)$ avoids the north pole of the Riemann sphere. (iv) For each point $\zeta$ in $N$ and each index $n$,

$$
\sigma\left(g_{n}(\zeta), \tilde{g}_{n}(\zeta)\right)<1 / n
$$

Condition (iv) ensures that for each point $\zeta$ in $N$ the two sequences $\left\{g_{n}(\zeta)\right\}$ and $\left\{\tilde{g}_{n}(\zeta)\right\}$ have the same set of cluster points. In the remainder of our proof, we assume without loss of generality that the original sequence $\left\{g_{n}\right\}$ satisfies conditions (i), (ii), and (iii), with the tildes deleted.

To use Lemma 6 in the proof of Lemma 7, we need an appropriate domain $G$, a relatively closed subset $K$ of $G$, and a function $h$ on $K$ whose behavior reflects in a suitable sense the behavior of the sequence $\left\{g_{n}\right\}$. Corresponding to each point $\zeta$ on $C$, we denote by $\varrho(\zeta)$ the ray $\{r \zeta: 0 \leq r<\infty\}$; similarly, corresponding to each set $M$ on $C$ we write

$$
\varrho(M)=\bigcup_{\varrho \in M} \varrho(\zeta)
$$


We now define the set $G$ and its subset $K$ by the formulas

$$
G=\Delta \cup \varrho(C \backslash N) \quad \text { and } \quad K=\Delta \cap \varrho(N) .
$$

Because $N$ is closed, $G$ is a domain, and because $N$ is nowhere-dense on $C$, the set $K$ is nowhere-dense in $G$; also, $K$ is closed relative to $G$, and the set $G^{\infty} \backslash K$ is connected and locally connected.

We begin the construction of the function $h$ by choosing for $h(0)$ the constant value of $g_{1}$. To construct $h$ on the remainder of the set $K$, we recall that $K$ is the union of the radial segments $[0, \zeta)(\zeta \in N)$. Corresponding to each $\zeta$ in $N$ and each index $n(n=1,2, \ldots)$, we require the function $h$ to effect the Möbius mapping of the segment $[(1-1 / n) \zeta,(1-1 /(n+1)) \zeta]$ onto the circular arc in C whose stereographic projection into the Riemann sphere is the geodesic joining the stereographic projections of the two points $g_{n}(\zeta)$ and $g_{n+1}(\zeta)$. Because the set $N$ is closed and nowhere dense on $C$, the function $h$ is continuous on $K$. The condition $g_{1} \neq g_{2}$ guarantees that $h$ is not constant.

If we define the function $\varepsilon$ on $K$ by the formula

$$
\varepsilon(z)=(1-|z|) \sigma_{0},
$$

and if $f$ is an analytic function in $G$ with the property described in Lemma 6, then $f$ is not constant, and for each point $\zeta$ in $N$ the radial cluster set of $f$ at $\zeta$ contains the set of cluster points of the sequence $\left\{g_{n}(\zeta)\right\}$. If in addition $\lim _{n \rightarrow \infty} \sigma\left[g_{n}(\zeta), g_{n+1}(\zeta)\right]=0$, the radial cluster set of $f$ at $\zeta$ coincides with the set of cluster points of $\left\{g_{n}(\zeta)\right\}$. This completes the proof of Lemma 7 .

Part (i) of Lemma 7 easily leads to a proof of the following.

Corollary. If $N$ is a nonempty, closed, nowhere-dense set on $C$ and $g$ is a mapping of $C$ into $\hat{\mathbf{C}}$, then the restriction $\left.g\right|_{N}$ is of Baire class 1 (in other words, is the limit of a pointwise convergent sequence of continuous functions) if and only if some function $f$ analytic on $\bar{\Delta} \backslash N$ satisfies at each point $\zeta$ in $N$ the condition $g(\zeta)=f^{*}(\zeta)$

In passing, we note that a modification of the proof of Lemma 7 enables us to prove a related result: if $g$ is a Baire-class-1 mapping of $C$ into $\hat{\mathbf{C}}$, then there exists an analytic function $f$ on $\Delta$ such that the radial-limit function $f^{*}$ agrees with $g$ on a prescribed first-category set (of measure $2 \pi$ ). However, considerations of zero-sets show that it is not possible to ensure that $f^{*}$ is defined at every point of $C$, or even on a subset of second category.

Next we prove a supplement to Theorem 1.

Theorem 1'. If $E$ is an uncountable analytic set on $C$ and $W$ is a (bounded) analytic set in $\hat{\mathbf{C}}$, then there exists a (bounded) analytic function $f$ on $\Delta$ whose radial limit exists at each point of $C$, with $U\left(f^{*}\right)=W$ and $U\left(\left.f^{*}\right|_{C \backslash E}\right)=\emptyset$. 
First we prove the theorem without the hypothesis that the set $W$ is bounded. By Lemma 3 , the set $E$ contains a perfect nowhere-dense set $P$. By Lemma 4, there exists a continuous function $g: P \rightarrow C$ such that $U(g)=W$. The corollary of Lemma 7 implies the existence of a nonconstant function $f$, analytic at each point of $\bar{\Delta} \backslash P$, having a radial limit at each point of $C$, and satisfying the condition $f^{*}(\zeta)=g(\zeta)$ everywhere on $P$. Because $f$ is analytic on $C \backslash P$ and is not constant, the inverse image $\left(\left.f^{*}\right|_{C \backslash P}\right)^{-1}(w)$ is countable, for each $w$ in $\hat{\mathbf{C}}$. It follows that $U\left(\left.f^{*}\right|_{C \backslash E}\right)=\emptyset$. The behavior of $f$ on radii ending in $P$ implies that $U\left(f^{*}\right)=U(g)=W$.

Late addition: F. Bagemihl and W. Seidel [Some boundary properties of analytic functions, Math. Z. 61, 1954, 186-199; Math. Rev. 16, p. 460; see Theorem 11, p. 198] proved our Theorem $1^{\prime}$ without its parenthetic restriction to bounded sets $W$ and bounded functions $f$.

To prove the theorem under the hypothesis that the set $W$ is bounded, we proceed as before, except for two alterations: we require the perfect set $P$ in $E$ to have measure 0 , and we replace the corollary of Lemma 7 with the following version of a result of Rudin [18].

Lemma 8. Let $g$ be a continuous, complex-valued function defined on a nonempty, closed set $N$ of measure 0 on the circle $C$. Then there exists a nonconstant, bounded function $f$, continuous on $\bar{\Delta}$ and analytic on $\bar{\Delta} \backslash N$, such that $\left.f\right|_{N}=g$.

Rudin's result is usually stated without the requirement that $f$ be analytic on $C \backslash N$ and not constant. To bridge the gap between the standard version and Lemma 8 , let $G$ denote a starlike domain containing the disk $\Delta$ and having a boundary that is the image of $C$ under a mapping $\gamma$ with the three properties

(i) $\gamma(\zeta)=\zeta$ if $\zeta \in N$,

(ii) $\gamma(\zeta) /|\gamma(\zeta)|=\zeta$ and $|\gamma(\zeta)|>1$ if $\zeta \in C \backslash N$,

(iii) $\gamma$ has a continuous second derivative with respect to arclength on $C$.

Let $\varphi$ denote a conformal mapping of $\Delta$ onto $G$, together with its continuous extension to $\bar{\Delta}$. By a theorem of O.D. Kellogg (see [8] or [21, p. 361]), the radiallimit function $\varphi^{*}$ has a nonvanishing second derivative with respect to arclength. In particular, because the set $N$ on $C$ has measure 0 , its inverse image $\varphi^{-1}(N)$ also has measure 0 . Now let $\zeta_{0}$ denote the midpoint of a component of $C \backslash \varphi^{-1}(N)$ having maximum length. Obviously, we can extend the function $g \circ \varphi$ to the set $\varphi^{-1}(N) \cup\left\{\zeta_{0}\right\}$ so that it is not constant. By the standard version of Rudin's theorem, there exists a function $h$, analytic on $\Delta$ and continuous on $\bar{\Delta}$ such that $h(\zeta)=g \circ \varphi(\zeta)$ for each $\zeta$ in $\varphi^{-1}(N) \cup\left\{\zeta_{0}\right\}$. The restriction $f$ to $\Delta$ of the function $h \circ \varphi^{-1}$ has the required properties.

This completes the proof of Lemma 8, and the lemma in turn completes the proof of Theorem $1^{\prime}$ and with it the proof of Theorem 1 . 
We note that for the case where the analytic set $W$ is bounded, we can choose the function $f$ in Theorem $1^{\prime}$ from any class of bounded analytic functions for which a suitable analogue of Lemma 8 is available. For example, a theorem of V.V. Peller and S.V. Khrushchëv [14] (with a modification similar to that in Rudin's result) allows us to use functions having a finite Dirichlet integral over $\Delta$.

Our method also permits the following extension of Lemma 4: If $E$ and $W$ are analytic sets in $\hat{\mathbf{C}}$ and $E$ is uncountable, then there exists a continuous function $g: \hat{\mathbf{C}} \rightarrow \hat{\mathbf{C}}$ such that $U(g)=W$ and the uncountable-order set of the restriction of $g$ to $\hat{\mathbf{C}} \backslash E$ is empty.

To prove this extension, let $N$ denote a nowhere-dense perfect subset of $E$ (Lemma 3 ensures the existence of such a subset), and let $\gamma$ denote a Jordan curve passing through each point of $N$. Then some homeomorphism $h$ of $\hat{\mathbf{C}}$ onto itself maps $\gamma$ onto $C$ (see [6, p. 155, Theorem 3]).

If $W$ is bounded, there exists a continuous function $f$ on $\bar{\Delta}$ such that $U(f)=$ $W$ and $U\left(\left.f\right|_{\Delta \backslash h(N)}\right)=\emptyset$ (see Theorem $1^{\prime}$ and its proof). Let

$$
g(z)= \begin{cases}f \circ h(z) & \text { if } \in \bar{\Delta} \\ f[h(1 / \bar{z})] & \text { if } z \in \hat{\mathbf{C}} \backslash \Delta\end{cases}
$$

with the convention that $1 / 0=\infty$. Then the function $g$ has the required properties.

We now sketch a proof of the extension for the case where the set $W$ is unbounded; it requires the construction of the quotient $g=g_{1} / g_{2}$ of two bounded functions. To achieve this, we first observe that we can modify the proof of Theorem $1^{\prime}$ to show that if $W^{*}$ is a bounded analytic set in $\hat{\mathbf{C}}$ and $P_{1}$ and $P_{2}$ are disjoint perfect sets of measure 0 on $C$, then there exists a function $f$, continuous on $\bar{\Delta}$ and analytic in $\bar{\Delta} \backslash\left(P_{1} \cup P_{2}\right)$, such that $f(\zeta)=1$ for all $\zeta$ in $P_{2}$ and the uncountable-order sets of the restrictions of $f$ to $P_{1}$ and $\bar{\Delta} \backslash P_{2}$ coincide with $W^{*}$.

In particular, let $P_{1}$ and $P_{2}$ be disjoint perfect subsets of measure 0 of the set $h(N)$ on $C$ (see the first paragraph after the statement of the extension). Let the relation of $g_{1}$ to $f$ be similar to the relation of $g$ to $f$ in the modified version of Theorem 1' except that the role of the bounded set $W^{*}$ in the modified version is played by the bounded set $W \cap \Delta$. Let $g_{2}$ be chosen similarly, except that the roles of $P_{2}$ and $P_{1}$ are reversed and $W^{*}$ is now replaced with the set $\{1 / z: z \in W \backslash \bar{\Delta}\}$. Then the function $g=g_{1} / g_{2}$ has the required properties.

We close this section by noting that Theorem 2 follows directly from Lemma 7 and the modified result of Hahn stated at the end of Section 2.

\section{Blaschke factors and Blaschke products}

In this section, we prove two lemmas that will provide technical support for our construction in Section 5. 
The Blaschke factor corresponding to a point $a$ in $\bar{\Delta}$ is the function

$$
b(z, a)= \begin{cases}z & \text { if } a=0 \\ (\bar{a} /|a|)(a-z) /(1-\bar{a} z)=|a|^{-1}(a-z)(1 / \bar{a}-z)^{-1} & \text { if } a \neq 0\end{cases}
$$

If $a \in C$, then $1 / \bar{a}=a$, and therefore the function $b(z, a)$ degenerates to the constant 1. In Section 5, it will be convenient to admit degenerate Blaschke factors into the construction.

A sequence $\left\{a_{k}\right\}$ in $\bar{\Delta}$ is a Blaschke sequence if it satisfies the Blaschke condition $\sum\left(1-\left|a_{k}\right|\right)<\infty$. Each finite or infinite Blaschke sequence $\left\{a_{k}\right\}$ in $\bar{\Delta}$ determines a Blaschke product

$$
B(z)=B\left(z,\left\{a_{k}\right\}\right)=\prod_{k} b\left(z, a_{k}\right) .
$$

Every Blaschke product $B\left(z,\left\{a_{k}\right\}\right)$ is an inner function, and it has an analytic extension across each arc of the circle $C$ that contains no limit point of the sequence $\left\{a_{k}\right\}$. For basic information on Blaschke products, we refer the reader to $[3, \mathrm{pp} .28-35]$ and to [4].

Lemma 9. Let $\Gamma$ denote the intersection of $\bar{\Delta}$ with some circle whose center $\zeta$ lies on $C$ and whose radius is less than 1 . Then, as the point a describes the arc $\Gamma$ in the counterclockwise direction, the value of $b(\zeta, a)$ describes the circle $C$ from 1 to 1 , also in the counterclockwise direction.

To prove the lemma, we observe that inversion in $C$ maps the arc $\Gamma$ onto a circular arc $\Gamma^{\prime}$ such that the point $\zeta$ lies inside the Jordan curve $\Gamma \cup \Gamma^{\prime}$. If $a$ is one of the endpoints of $\Gamma$, the function $b(z, a)$ is the constant 1 . As the point $a$ moves counterclockwise along $\Gamma$, from $C$ to $C$, the two quantities

$$
\arg (a-\zeta) \quad \text { and } \quad-\arg (1 / \bar{a}-\zeta)
$$

increase, the first by some quantity $\theta(0<\theta<\pi)$, the other by $2 \pi-\theta$. This concludes the proof.

Our proof of Lemma 9 makes it evident that if $0<|a|<1$ and $|\zeta|=1$, and if the distance $|a-\zeta|$ is small, then $\arg b(\zeta, a)$ is approximately twice the angle from the half-tangent to $C$ at $\zeta$ to the vector $a-\zeta$.

Lemma 10. For every Blaschke sequence $\left\{a_{k}\right\}$ the inequality

$$
\left|1-B\left(z,\left\{a_{k}\right\}\right)\right|<2 \sum \frac{1-\left|a_{k}\right|}{\left|a_{k}-z\right|}
$$

holds at each point $z$ in $\Delta$. 
First we prove the auxiliary inequality

$$
|1-b(z, a)| \leq 2 \frac{1-|a|}{|a-z|}
$$

If $a=0$, then $|1-b(z, a)|=|1-z| \leq 2 /|z|$ for all $z$ in $\bar{\Delta}$. If $a \neq 0$, we write $a=\varrho e^{i \theta}$ and $z=r e^{i(\theta+\lambda)}$. Then

$$
b(z, a)=\frac{\varrho-r e^{i \lambda}}{1-\varrho r e^{i \lambda}}
$$

and therefore

$$
1-b(z, a)=\frac{(1-\varrho)\left(1+r e^{i \lambda}\right)}{1-\varrho r e^{i \lambda}}=\frac{1-|a|}{a-z} \frac{a-z}{1-\bar{a} z}\left(1+r e^{i \lambda}\right) .
$$

The third factor in the last member has modulus at most $1+r$, and the second factor has modulus $|b(z, a)|$. Therefore the auxiliary inequality holds everywhere in $\bar{\Delta}$.

Finally, we consider the identity

$$
\begin{aligned}
1-\prod_{1}^{n} b\left(z, a_{k}\right)= & 1-b\left(z, a_{1}\right)+\left(1-b\left(z, a_{2}\right)\right) b\left(z, a_{1}\right)+\cdots \\
& +\left(1-b\left(z, a_{n}\right)\right) \prod_{1}^{n-1} b\left(z, a_{k}\right),
\end{aligned}
$$

recall that $\left|\prod_{1}^{m} b\left(z, a_{k}\right)\right| \leq 1$ for each $m$, and complete the proof by applying the triangle inequality and our auxiliary inequality.

\section{Special Blaschke products}

This section's central result, Lemma 11, is an analogue for Blaschke products of Lemma 7 in Section 3. It will serve in the proofs of Theorems 3 and 4.

Lemma 11. Corresponding to every uncountable analytic set $E$ on the circle $C$, there exist a nonempty perfect subset $P$ of $E$ and a Blaschke product $\tilde{B}$, the latter analytic on $\bar{\Delta} \backslash P$, such that if $\left\{g_{n}\right\}$ is a sequence of continuous mappings of $P$ into $C$, then some subproduct $B$ of $\tilde{B}$ has at each point $\zeta$ of $P$ the following properties: the radial cluster set of $B$ lies on $C$ and contains the set of subsequential limit points of the sequence $\left\{g_{n}(\zeta)\right\}$; moreover, in case $\lim _{n \rightarrow \infty}\left(g_{n}(\zeta)-g_{n+1}(\zeta)\right)=0$, the two point sets coincide. 
By Lemma 3 of Section 2, we can assume without loss of generality that the set $E$ is perfect and nowhere dense. From $E$ we shall now select a perfect set $P$ thin enough to permit the construction of the master-function $\tilde{B}$ promised in Lemma 11. Our procedure will lead to a natural representation $P=\left\{\zeta_{\alpha}\right\}$, where the index $\alpha$ ranges over the set of all infinite dyadic sequences, that is, all infinite sequences of 0 's and 1 's.

At the $m$ th stage of the construction of $P$, we shall select a set of $2^{m}$ points in $E$. We shall denote the points by $\zeta_{\beta}$ or $\zeta_{\gamma}$, where the symbols $\beta$ and $\gamma$ range over the $2^{m}$ dyadic sequences of length $|\beta|=m$ or $|\gamma|=m$. Our choice of these points will guarantee that if $\alpha$ is an infinite dyadic sequence and if for $m=1,2, \ldots$ the symbol $\alpha(m)$ denotes the $m$-element initial segment of $\alpha$, then the sequence $\left\{\zeta_{\alpha(m)}\right\}$ converges; its limit point will serve as the point $\zeta_{\alpha}$ in the set $P$.

The placement of the zeros of the master function $\tilde{B}$ is based on a simple geometric procedure. Corresponding to each point $\zeta$ on $C$ and each number $\delta$ $(0<\delta<1)$, we denote by $d(\zeta, \delta)$ and $c(\zeta, \delta)$ the intersection of $\bar{\Delta}$ with the disk $\{z:|z-\zeta|<\delta\}$ and the circle $\{z:|z-\zeta|=\delta\}$, respectively.

Corresponding to the dyadic one-element sequences $\beta=\{0\}$ and $\beta=\{1\}$, we choose any two distinct points $\zeta_{\beta}(|\beta|=1)$ in $E$. In the interval $\left(0, e^{-3}\right)$ we choose a number $\delta_{1}$ small enough so that the two $\operatorname{arcs} c\left(\zeta_{\beta}, \delta_{1}\right)(|\beta|=1)$ are disjoint. On each of the two corresponding shorter $\operatorname{arcs} c\left(\zeta_{\beta}, \delta_{1}^{2}\right)$ we assign to the master-product $\tilde{B}$ exactly two zeros $a_{\beta h}(h=1,2)$ in such a way that the corresponding Blaschke factors $b_{\beta h}$ satisfy the condition $b_{\beta h}\left(\zeta_{\beta}\right)=\exp 2 \pi i h / 2$. (This is possible, by Lemma 9.)

Suppose that for each of the indices $k=1,2, \ldots, m-1$ we have chosen $2^{k}$ points $\zeta_{\beta}(|\beta|=k)$ in $E$, together with a positive number $\delta_{k}$, and that each $\delta_{k}$ is small enough so that the $2^{k} \operatorname{arcs} c\left(\zeta_{\beta}, \delta_{k}\right)(|\beta|=k)$ are disjoint. Suppose also that corresponding to each $\beta(|\beta|=k)$ we have selected as zeros the $2^{k}$ points

$$
a_{\beta h} \quad\left(h=1,2, \ldots, 2^{k}\right)
$$

on $c\left(\zeta_{\beta}, \delta_{k}^{2}\right)$ in such a way that the corresponding Blaschke factors $b_{\beta h}$ satisfy the condition

$$
b_{\beta h}\left(\zeta_{\beta}\right)=\exp 2 \pi i h / 2^{k} .
$$

Let $\gamma$ denote one of the $2^{m}$ dyadic sequences of length $m$, and let $\beta$ be its $(m-1)$-element initial segment. For $\zeta_{\gamma}$ we choose the point $\zeta_{\beta}$ if the $m$ th element of $\gamma$ is 0 , a different point of $E$ on the boundary of $d\left(\zeta_{\beta}, \delta_{m-1}^{3}\right)$ if the $m$ th element of $\gamma$ is 1 . We then choose the number $\delta_{m}$ small enough so that the $2^{m}$ $\operatorname{arcs} c\left(\zeta_{\gamma}, \delta_{m}\right)(|\gamma|=m)$ are disjoint. On each of the $2^{m} \operatorname{arcs} c\left(\zeta_{\gamma}, \delta_{m}^{2}\right)(|\gamma|=m)$ we assign to the master-product $\tilde{B}$ exactly $2^{m}$ zeros $a_{\gamma h}\left(h=1,2, \ldots, 2^{m}\right)$ in such a way that the corresponding Blaschke factors $b_{\gamma h}$ satisfy the condition

$$
b_{\gamma h}\left(\zeta_{\gamma}\right)=\exp 2 \pi i h / 2^{m} .
$$


By virtue of our construction, $2 \delta_{m}<\delta_{m-1}^{3}$. Together with the restriction $\delta_{1}<e^{-3}$, this implies that

$$
\begin{aligned}
\log \delta_{2} & <3 \log \delta_{1}-\log 2 \\
\log \delta_{3} & <3 \log \delta_{2}-\log 2<9 \log \delta_{1}-(1+3) \log 2 \\
\vdots & \\
\log \delta_{m} & <-3^{m}-\left(1+3+\cdots+3^{m-2}\right) \log 2
\end{aligned}
$$

in other words, that

$$
\delta_{m}<2^{-\left(3^{m-1}-1\right) / 2} \exp \left(-3^{m}\right)<\exp \left(-3^{m}\right) .
$$

Because $2 m-\left(3^{m-1}-1\right) / 2 \leq 3$ when $m=1,2, \ldots$, we can also assert that

$$
\left(2^{m}\right)^{2} \delta_{m} \leq 8 \exp \left(-3^{m}\right) .
$$

Corresponding to each index $m$, our master-product $\tilde{B}$ has $2^{m}$ zeros on each of the $2^{m} \operatorname{arcs} c\left(\zeta_{\beta}, \delta_{m}^{2}\right)(|\beta|=m)$, and each of these zeros lies in the annulus $\left\{1 \geq|z| \geq 1-\delta_{m}^{2}\right\}$. Because

$$
\sum_{1}^{\infty}\left(2^{m}\right)^{2} \delta_{m}^{2}<\infty
$$

the product $\tilde{B}$ is a genuine Blaschke product. Moreover, the set of limit points of the set of its zeros coincides with the set $P$. Therefore the product $\tilde{B}$, together with all its subproducts, is analytic on $C \backslash P$.

Our next step is to construct, corresponding to each sequence $\left\{g_{n}\right\}$ of continuous mappings of $P$ into $C$, a subproduct $B$ of $\tilde{B}$. Our product $B$ will have the desired properties, provided $g_{n+1}(\zeta)-g_{n}(\zeta) \rightarrow 0$ uniformly on $P$, as $n \rightarrow \infty$, and provided the maximum $\omega\left(2 \delta_{m}^{3}, g_{n}\right)$ of the quantity $\left|g_{n}(\zeta)-g_{n}\left(\zeta^{\prime}\right)\right|$, taken over all point pairs $\left(\zeta, \zeta^{\prime}\right)$ in $P \times P$ for which $\left|\zeta-\zeta^{\prime}\right| \leq 2 \delta_{m}^{3}$, tends to 0 as $n \rightarrow \infty$.

Not all admissible sequences $\left\{g_{n}\right\}$ satisfy these conditions; but we shall show how to embed every admissible sequence in an expanded sequence that satisfies the two conditions and is for our purpose equivalent to the original sequence.

Let $B_{0}$ denote the constant function whose value is 1 . Suppose $m$ is a positive integer and we have constructed a finite subproduct $B_{m-1}$ of $\tilde{B}$ whose zeros lie on the $\operatorname{arcs} c\left(\zeta_{\beta}, \delta_{k}^{2}\right)(|\beta|=k ; k=1,2, \ldots, m-1$; exactly one zero on each of the arcs).

Corresponding to each dyadic sequence $\gamma(|\gamma|=m)$ we choose for $b_{\gamma}$ one of the $2^{m}$ simple Blaschke factors $b_{\gamma h}$ of $\tilde{B}$ with a zero on the $\operatorname{arc} c\left(\zeta_{\gamma}, \delta_{m}^{2}\right)$. More 
precisely, we choose one of the factors $b_{\gamma h}$ that minimize the quantity $\mid g_{m}\left(\zeta_{\gamma}\right)-$ $B_{m-1}\left(\zeta_{\gamma}\right) b_{\gamma h}\left(\zeta_{\gamma}\right) \mid$ (there are at most two), and we observe that by virtue of our choice of the points $a_{\gamma h}\left(h=1,2, \ldots, 2^{|\gamma|}\right)$, the factor $b_{\gamma}$ we have chosen satisfies the condition

$$
\left|g_{m}\left(\zeta_{\gamma}\right)-B_{m-1}\left(\zeta_{\gamma}\right) b_{\gamma h}\left(\zeta_{\gamma}\right)\right| \leq \pi 2^{-|\gamma|} .
$$

We define the provisional finite product

$$
B_{m}=B_{m-1} \prod_{|\gamma|=m} b_{\gamma}
$$

and the possibly unsatisfactory infinite product $B=\lim _{m \rightarrow \infty} B_{m}$.

To see that the Blaschke product $B$ does not necessarily have the desired properties, suppose for example that for each positive integer $n$ and each dyadic sequence $\beta$ of length $n$ the function $g_{n}$ satisfies the condition $g_{n}\left(\zeta_{\beta}\right)=(-1)^{n}$. Then, for each dyadic sequence $\beta$ of finite length, the value of the factor $b_{\beta}$ of $B$ at $\zeta_{\beta}$ must lie near -1 , and therefore the zero $a_{\beta}$ of $b_{\beta}$ must lie near the midpoint of the arc $c\left(\zeta_{\beta}, \delta_{n}^{2}\right)$. Consequently, at each point $\zeta$ of $P$ the radial cluster set of $B$ contains the point 0 , even if $\lim _{n \rightarrow \infty} g_{n}(\zeta)$ exists.

We overcome this difficulty by inserting certain additional functions into the sequence $\left\{g_{n}\right\}$. To choose the functions to be inserted between the elements $g_{n}$ and $g_{n+1}(n=1,2, \ldots)$, we divide the circle $C$ into finitely many $\operatorname{arcs} C_{n h}$ $\left(h=1,2, \ldots, H_{n}\right)$ whose endpoints lie in $C \backslash P$. Because $g_{n}$ and $g_{n+1}$ are uniformly continuous on $P$, we can choose the $\operatorname{arcs} C_{n h}$ short enough so that for each index $h$ the images $g_{n}\left(C_{n h} \cap P\right)$ and $g_{n+1}\left(C_{n h} \cap P\right)$ have diameter less than 1. Because for each of the indices $h=1,2, \ldots, H_{n}$ the complement of the set

$$
g_{n}\left(C_{n h} \cap P\right) \cup g_{n+1}\left(C_{n h} \cap P\right)
$$

then contains an arc on $C$ of length greater than $2 \pi / 3$, there exists an interval $I_{n h}$ of length less than $4 \pi / 3$ and with the property that we can regard the restrictions to $C_{n h} \cap P$ of the two functions

$$
\varphi_{n}=\arg g_{n} \quad \text { and } \quad \varphi_{n+1}=\arg g_{n+1}
$$

as continuous functions whose range lies in $I_{n h}$. For each of the indices $j=$ $0,1, \ldots, n-1$ we define the function $g_{n j}$ on $C_{n h} \cap P$ by the formula

$$
g_{n j}=\exp \left(i\left((n-j) \arg g_{n}+j \arg g_{n+1}\right) / n\right),
$$

and we replace the element $g_{n}$ in $\left\{g_{n}\right\}$ with the finite sequence

$$
\left\{g_{n 0}, g_{n 1}, \ldots, g_{n, n-1}\right\} \text {. }
$$


For the sake of clerical convenience, we revert to single indices; that is, we now let the symbol $\left\{g_{n}\right\}$ represent the sequence

$$
\left\{g_{10}, g_{20}, g_{21}, g_{30}, g_{31}, g_{32}, g_{40}, \ldots\right\} \text {. }
$$

From the formula $\sum_{j=1}^{n} j=n(n+1) / 2$ we see that at each point $\zeta$ in $P$ the new sequence satisfies the inequality

$$
\left|g_{n}(\zeta)-g_{n+1}(\zeta)\right|<2 \pi / \sqrt{n}
$$

In our proof of Lemma 11, we shall also need the hypothesis that $\omega\left(2 \delta_{m}^{3}, g_{m}\right)$ $\rightarrow 0$ as $m \rightarrow \infty$. To modify $\left\{g_{n}\right\}$ so that this condition is satisfied, we replace each element $g_{n}$ with a finite block that repeats the element $g_{n}$ so many times that if the element $g_{n+1}$ first appears in the $m$ th position, the corresponding number $\delta_{m}$ is small enough to guarantee that

$$
\omega\left(2 \delta_{m-1}^{3}, g_{n+1}\right) \leq 2 \pi /(n+1) .
$$

We again supply new indices, and we denote the modified sequence by $\left\{g_{n}\right\}$.

Because the sequence $\left\{g_{n}\right\}$ is now adjusted to the constraints arising from properties of the sequence $\left\{\delta_{m}\right\}$-which in turn reflect geometric properties of the set $E$-we can safely replace the letter $n$ in $\left\{g_{n}\right\}$ with the letter $m$. We can not make the corresponding replacement in the numerical bounds achieved in the two preceding paragraphs; but we can assert that there exists a sequence $\left\{\varepsilon_{m}\right\}$ such that

$$
\left|g_{m}(\zeta)-g_{m+1}(\zeta)\right|<\varepsilon_{m} \quad(\zeta \in P)
$$

$$
\omega\left(2 \delta_{m-1}^{3}, g_{m}\right)<\varepsilon_{m},
$$

and $\varepsilon_{m} \rightarrow 0$ as $m \rightarrow \infty$. How slowly $\varepsilon_{m} \rightarrow 0$ depends on the sequence $\left\{\delta_{n}\right\}$ and certain qualities of the original sequence $\left\{g_{n}\right\}$. We do not attempt a quantitive description of the sequence $\left\{\varepsilon_{m}\right\}$, for such a description would not simplify the computations that are still necessary in our proof of Lemma 11.

It remains to show that after the imposition of our two modifications the sequence $\left\{g_{m}\right\}$ generates a subproduct $B$ of $\tilde{B}$ with the properties described in Lemma 11. Since the original sequence is a subsequence of the modified sequence, the set of limit points of the new sequence $\left\{g_{m}(\zeta)\right\}$ includes (for each $\zeta$ in $P$ ) the set of limit points of the original sequence $\left\{g_{n}(\zeta)\right\}$; inspection shows that the two sets coincide if the original sequence satisfies the condition $g_{n}(\zeta)-g_{n+1}(\zeta) \rightarrow 0$.

With each positive integer $k$ we associate the set

$$
D_{k}=\bigcup_{|\beta|=k} d\left(\zeta_{\beta}, \delta_{k}\right)
$$


and corresponding to each finite dyadic sequence $\beta$ we define the sector

$$
S_{\beta}=\left\{z:\left|\arg \left(z / \zeta_{\beta}\right)\right|<2 \delta_{|\beta|}^{3},|z|<1\right\} .
$$

If $\alpha$ is an infinite dyadic sequence and $\beta=\beta(\alpha, m)$ is its $m$-element initial section, we write

$$
S^{*}(\alpha, m)=S_{\beta}^{*}=S_{\beta} \cap d\left(\zeta_{\beta}, \delta_{m}\right) \backslash D_{m+1} .
$$

Clearly, $S_{\beta}^{*}$ consists of a terminal portion of $S_{\beta}$ minus two nearly complete semidisks of radius $\delta_{|\beta|+1}$. Because $\delta_{1}<e^{-3}$ and

$$
\delta_{m}^{3}+\delta_{m+1}^{3}+\cdots<2 \delta_{m}^{3},
$$

$\zeta_{\alpha}$ lies on the boundary of $d\left(\zeta_{\beta}, 2 \delta_{m}^{3}\right)$ and each point of each radial segment $\left(\left(1-\delta_{1} / 2\right) \zeta_{\alpha}, \zeta_{\alpha}\right)$ lies in one of the regions $S_{\beta}^{*}(|\beta|=1,2, \ldots)$.

The proof of Lemma 11 will therefore be complete when we have shown that for each $\alpha$ the supremum of the quantities

$$
\left\{\left|g_{m}\left(\zeta_{\alpha}\right)-B(z)\right|: z \in S^{*}(\alpha, m)\right\}
$$

tends to 0 as $m \rightarrow \infty$. By the triangle inequality,

$$
\begin{aligned}
\left|g_{m}\left(\zeta_{\alpha}\right)-B(z)\right| \leq & \left|g_{m}\left(\zeta_{\alpha}\right)-g_{m}\left(\zeta_{\beta}\right)\right|+\left|g_{m}\left(\zeta_{\beta}\right)-B_{m}\left(\zeta_{\beta}\right)\right| \\
& +\left|B_{m}\left(\zeta_{\beta}\right)-B_{m}(z)\right|+\left|B_{m}(z)-B(z)\right| \\
= & e_{1}+e_{2}+e_{3}+e_{4} .
\end{aligned}
$$

Because $\left|\zeta_{\alpha}-\zeta_{\beta}\right|<2 \delta_{m-1}^{3}$, it follows from the relation $(* * *)$ that $e_{1}<\varepsilon_{m}$, and therefore $e_{1} \rightarrow 0$ as $m \rightarrow \infty$.

To estimate the term $e_{2}$, we note that

$$
\begin{aligned}
\left|g_{m}\left(\zeta_{\beta}\right)-B_{m}\left(\zeta_{\beta}\right)\right| \leq & \left|g_{m}\left(\zeta_{\beta}\right)-B_{m-1}\left(\zeta_{\beta}\right) b_{\beta}\left(\zeta_{\beta}\right)\right| \\
& +\left|B_{m-1}\left(\zeta_{\beta}\right) b_{\beta}\left(\zeta_{\beta}\right)\left(1-B_{m}\left(\zeta_{\beta}\right) / B_{m-1}\left(\zeta_{\beta}\right) b_{\beta}\left(\zeta_{\beta}\right)\right)\right| .
\end{aligned}
$$

By virtue of the inequality $(*)$ and our choice of the zero $a_{\beta}$ of the Blaschke factor $b_{\beta}$, the first term on the right is not greater than $\pi 2^{-m}$. In the second term, each of the first two factors has modulus 1 , and the third factor is

$$
\left|1-\prod_{\substack{|\gamma|=m \\ \gamma \neq \beta}} b_{\gamma}\left(\zeta_{\beta}\right)\right| .
$$

If $|\gamma|=m$, then $1-\left|a_{\gamma}\right| \leq \delta_{m}^{2}$; if in addition $\gamma \neq \beta$, then $\left|\zeta_{\beta}-a_{\gamma}\right|>\delta_{m}$. It follows from Lemma 10 that the third factor in the second term is less than

$$
2^{m+1} \delta_{m}^{2} / \delta_{m}=2^{m+1} \delta_{m}
$$


Therefore $e_{2} \rightarrow 0$ as $m \rightarrow \infty$.

Our treatment of $e_{3}$ is based on the two identities

$$
B_{m}(z)-B_{m}\left(\zeta_{\beta}\right)=\int_{\zeta_{\beta}}^{z} B_{m}^{\prime}(w) d w
$$

and

$$
B_{m}^{\prime}=\sum_{|\gamma| \leq m}\left(B_{m} / b_{\gamma}\right) b_{\gamma}^{\prime}
$$

Using as path of integration the rectilinear segment $\sigma=\left[\zeta_{\beta}, z\right]$ and observing that each of the coefficients $B_{m} / b_{\gamma}$ is a Blaschke product, we see that

$$
e_{3} \leq \sum_{|\gamma| \leq m} \int_{\sigma}\left|b_{\gamma}^{\prime}(w)\right||d w|=\sum_{1}+\sum_{2}+\sum_{3}
$$

here we use the symbol $\sum_{1}$ for the sum of the terms in which the index $\gamma$ has length less than $m$, the symbol $\sum_{2}$ for the sum of the terms in which $|\gamma|=m$ and $\gamma \neq \beta$; the symbol $\sum_{3}$ represents the single term $\int_{\sigma}\left|b_{\beta}^{\prime}\right||d w|$.

If $\left|a_{\gamma}\right|=1$, then $b_{\gamma}^{\prime}$ is the constant function 0 . If $\left|a_{\gamma}\right|<1$, then

$$
\left|b_{\gamma}^{\prime}(w)\right|=\left(1-\left|a_{\gamma}\right|^{2}\right) /\left|1-\bar{a}_{\gamma} w\right|^{2},
$$

and therefore the maximum modulus of $b_{\gamma}^{\prime}$ in the closure of $\Delta$ is $\left(1+\left|a_{\gamma}\right|\right)$ $/\left(1-\left|a_{\gamma}\right|\right)$; clearly, this is less than $2 /\left(1-\left|a_{\gamma}\right|\right)$. To obtain a positive lower bound on $1-\left|a_{\gamma}\right|$ for the cases where $\left|a_{\gamma}\right|<1$, we use the identity

$$
1-b(\zeta, a)=\frac{1-|a|}{a-\zeta} \cdot \frac{a-\zeta}{1-\bar{a} \zeta} \cdot\left(1+r e^{i \lambda}\right)
$$

from the proof of Lemma 10. The second factor on the right has modulus 1 when $\zeta \in C$, and the third factor has modulus less than 2 . It follows that

$$
1-\left|a_{\zeta}\right|>\left|1-b_{\gamma}\left(\zeta_{\gamma}\right)\right|\left|a_{\gamma}-\zeta_{\gamma}\right| / 2 .
$$

Because $b_{\gamma}\left(\zeta_{\gamma}\right)$ is one of the $2^{|\gamma|}-1$ numbers

$$
\exp \left(h \pi i 2^{1-|\gamma|}\right) \quad\left(h=1,2, \ldots, 2^{|\gamma|}-1\right)
$$

and $a_{\gamma}$ lies on the $\operatorname{arc} c\left(\zeta_{\gamma}, \delta_{|\gamma|}^{2}\right)$, it follows further that

$$
1-\left|a_{\gamma}\right|>(2 / \pi) 2 \pi \cdot 2^{-|\gamma|} \delta_{|\gamma|}^{2} / 2=2^{1-|\gamma|} \delta_{|\gamma|}^{2}
$$


therefore $\left|b_{\gamma}^{\prime}(w)\right|<2 /\left(1-\left|a_{\gamma}\right|\right)<2^{|\gamma|} / \delta_{|\gamma|}^{2}$.

Since $z \in S_{\beta}^{*} \subset d\left(\zeta_{\beta}, \delta_{m}\right)$, the segment $\sigma$ has length at most $\delta_{m}$, and it follows that

$\sum_{1}<\delta_{m}\left(2+2^{2}+2^{4}+\cdots+2^{2 m-2}\right) / \delta_{m-1}^{2}<\left(\delta_{m-1}^{3} / 2\right) 2^{2 m-1} / \delta_{m-1}^{2}=2^{2 m-2} \delta_{m-1}$.

Clearly, $\sum_{1} \rightarrow 0$ as $m \rightarrow \infty$.

To estimate $\sum_{2}$, we observe first that each of the corresponding functions $b_{\gamma}$ maps the segment $\sigma$ one-to-one onto a circular arc. In each case, $1-\left|a_{\gamma}\right| \leq \delta_{m}^{2}$, and the distance between the points $a_{\gamma}$ and the segment $\sigma$ is greater than $\delta_{m}$. Therefore

$$
\left|1-b_{\gamma}(w)\right|<2 \delta_{m}^{2} / \delta_{m}=2 \delta_{m}
$$

in other words, the arc $b_{\gamma}(\sigma)$ lies in a circular disk of radius less than $2 \delta_{m}$. Its length (which is equal to the integral $\int_{\sigma}\left|b_{\gamma}^{\prime}\right||d w|$ ) is therefore less than $4 \pi \delta_{m}$, and consequently the sum of the $2^{m}-1$ integrals in $\sum_{2}$ is less than $2^{m+2} \pi \delta_{m}$. Again, this tends to 0 as $m \rightarrow \infty$.

Our estimate of $\sum_{3}$ requires additional information on the position of the zero $a_{\beta}$ of the Blaschke factor $b_{\beta}$, information equivalent to the assertion that $b_{\beta}\left(\zeta_{\beta}\right) \rightarrow 1$ as $|\beta| \rightarrow \infty$. Let $\eta$ denote the $(m-1)$-element initial segment of $\beta$. By the triangle inequality,

$$
\begin{aligned}
\mid g_{m}\left(\zeta_{\beta}\right) & -B_{m-1}\left(\zeta_{\beta}\right)|\leq| g_{m}\left(\zeta_{\beta}\right)-g_{m-1}\left(\zeta_{\beta}\right) \mid \\
& +\left|g_{m-1}\left(\zeta_{\beta}\right)-g_{m-1}\left(\zeta_{\eta}\right)\right|+\left|g_{m-1}\left(\zeta_{\eta}\right)-B_{m-2}\left(\zeta_{\eta}\right) b_{\eta}\left(\zeta_{\eta}\right)\right| \\
& +\left|B_{m-2}\left(\zeta_{\eta}\right) b_{\eta}\left(\zeta_{\eta}\right)-B_{m-1}\left(\zeta_{\eta}\right)\right|+\left|B_{m-1}\left(\zeta_{\eta}\right)-B_{m-1}\left(\zeta_{\beta}\right)\right| .
\end{aligned}
$$

By $(* *)$, the first term on the right is less than $\varepsilon_{m}$.

By $(* * *)$, the second term on the right is less than $\varepsilon_{m-1}$.

By our choice of the zero $a_{\eta}$ of the Blaschke factor $b_{\eta}$ (see $(*)$ ), the third term on the right is less than $\pi / 2^{m-1}$.

We can write the fourth term in the form

$$
\left|B_{m-2}\left(\zeta_{\eta}\right) b_{\eta}\left(\zeta_{\eta}\right)\right|\left|1-\prod_{\substack{|\gamma|=m-1 \\ \gamma \neq \eta}} b_{\gamma}\left(\zeta_{\eta}\right)\right| .
$$

The first factor is 1 , and by Lemma 10 the second factor is less than

$$
2 \cdot 2^{m-1} \delta_{m-1}^{2} / \delta_{m-1}=2^{m} \delta_{m-1} .
$$

The last of the five terms is less than

$$
\sum_{|\gamma| \leq m-1} \int_{\zeta_{\eta}}^{\zeta_{\beta}}\left|b_{\gamma}^{\prime}(w)\right||d w| .
$$


We integrate along the rectilinear segment $\left[\zeta_{\eta}, \zeta_{\beta}\right]$, and we note that this has length less than $\delta_{m-1}^{3}$. Again we use the inequality

$$
\left|b_{\gamma}^{\prime}(w)\right|<2^{|\gamma|} / \delta_{|\gamma|}^{2} .
$$

Corresponding to each of the index lengths $|\gamma|=1,2, \ldots, m-1$, our sum contains $2^{|\gamma|}$ integrals. Consequently, the final term on the right side of our inequality is less than

$$
\delta_{m-1}^{3}\left(2^{2}+2^{4}+\cdots+2^{2(m-1)}\right) / \delta_{m-1}^{2}<2^{2 m-1} \delta_{m-1} .
$$

We have shown that $g_{m}\left(\zeta_{\beta}\right)-B_{m-1}\left(\zeta_{\beta}\right) \rightarrow 0$ as $m=|\beta| \rightarrow \infty$. By virtue of $(*)$, this implies that $\arg b_{\beta}\left(\zeta_{\beta}\right) \rightarrow 0$ as $|\beta| \rightarrow \infty$, and from the comment after the proof of Lemma 9 it follows that $\left(1-\left|a_{\beta}\right|\right) / \delta_{|\beta|}^{2} \rightarrow 0$ as $|\beta| \rightarrow \infty$. By Lemma 10 , the maximum modulus of $1-b_{\beta}(w)$ on the segment $\sigma$ is small, and therefore $\sum_{3} \rightarrow 0$ as $m \rightarrow \infty$.

Finally,

$$
e_{4}=\left|B_{m}(z)\left(1-B(z) / B_{m}(z)\right)\right|=\left|B_{m}(z)\right|\left|1-\prod_{|\gamma|>m} b_{\gamma}(z)\right| .
$$

Because $\left|1-a_{\gamma}\right| \leq \delta_{|\gamma|}^{2}$ and $\left|z-a_{\gamma}\right|>\delta_{|\gamma|} / 2$ for all $z$ outside the set $D_{|\gamma|+1}$, it follows from Lemma 10 that

$$
e_{4}<\sum_{k>m} 2^{k+2} \delta_{k}
$$

This completes the proof of Lemma 11.

Theorem 3 is a direct consequence of Lemmas 4 and 11 (compare the proof of Theorem $1^{\prime}$ ). Theorem 4 follows from Lemma 11 and the result of Hahn stated at the end of Section 2.

The following is a corollary of Lemma 11.

Corollary. If $E$ is an uncountable analytic subset of $C$, then there exists a nonempty perfect subset $P$ of $E$ with the following property. For every function $g: C \rightarrow C$, the restriction $\left.g\right|_{P}$ of $g$ to $P$ is of Baire class 1 if and only if there exists a nonconstant Blaschke product $B$, analytic at each point of $\bar{\Delta} \backslash P$, such that $B^{*}(\zeta)=g(\zeta)$ for each $\zeta$ in $P$.

In analogy to the cardinality of the set on $C$ where the radial limit $f^{*}$ of a function $f$ on $\Delta$ has a specific value $w_{0}$, we can consider the cardinality of the set on $C$ where the radial cluster set of $f$ consists of a specific continuum $K_{0}$. Unfortunately, the space of continua in $\hat{\mathbf{C}}$ is the Hilbert cube. But the space of subcontinua of $C$ is topologically equivalent to $\bar{\Delta}$. Consequently, moderate changes in the proof of Theorem 3 will lead to the following proposition.

Theorem 3'. There exists a Blaschke product $\tilde{B}$ such that each analytic set $W$ of (possibly degenerate) arcs on $C$ is the uncountable-order set of radial cluster sets of some subproduct of $\tilde{B}$. 


\section{Positive-measure values and second-category values}

We shall establish an assertion slightly stronger than Theorem 5: To each countable set $W$ in $\hat{\mathbf{C}}$ there corresponds an analytic function $f$ on $\Delta$ such that $f^{*}$ exists everywhere on $C \backslash\{1\}$ and the set $\left\{\zeta: f^{*}(\zeta)=w\right\}$ has positive measure if $w \in W$ and is at most countably infinite if $w \notin W$.

If $W=\emptyset$ and $f(z)=z$ for all $z$, the function $f$ has the required properties. All other cases we treat simultaneously by regarding $W$ as an infinite sequence $\left\{w_{k}\right\}$ of finitely or infinitely many distinct values.

Corresponding to each positive integer $k$, we choose on the open arc

$$
\{\zeta: \zeta \in C, 1 /(k+1)<\arg \zeta<1 / k\}
$$

a closed, nowhere-dense set $N_{k}$ of positive measure. By $N$ we denote the closure of the union of the sets $N_{k}(k=1,2, \ldots)$. On $N$ we define the functions $h_{n}$ by the formula

$$
h_{n}(\zeta)= \begin{cases}w_{k} & \text { if } \zeta \in N_{k} \text { and } k=1,2, \ldots, n, \\ w_{n+1} & \text { if } \zeta \in \bigcup_{n+1}^{\infty} N_{k} \cup\{1\} .\end{cases}
$$

Obviously, each of the functions $h_{n}$ is the restriction to $N$ of a continuous function $g_{n}$ on $C$. By Lemma 7 , some function $f$ analytic on $\bar{\Delta} \backslash N$ satisfies at each point $\zeta$ of each of the sets $N_{k}$ the condition

$$
f^{*}(\zeta)=\lim _{n \rightarrow \infty} g_{n}(\zeta)=w_{k}
$$

This completes our proof.

We now turn to the proof of Theorem 6. Again, the function $f(z) \equiv z$ serves in the case where $W=\emptyset$. In the remaining cases, let $W=\left\{w_{n}\right\}$ denote a finite or infinite sequence of distinct points in $\hat{\mathbf{C}}$.

Let $E$ denote a residual set (that is, the complement of a first-category set) of measure 0 on the interval $(-\pi, 0)$, and let $\mu$ denote a nondecreasing function on $[-\pi, \pi]$, constant in $(0, \pi)$ and with $\mu^{\prime}=\infty$ everywhere in $E$ (see [15, p. 214]). Then the bounded analytic function

$$
g(z)=\exp \int_{-\pi}^{\pi} \frac{z+e^{i t}}{z-e^{i t}} d \mu(t)
$$

is nonvanishing on $\Delta$ and has the radial limit 0 everywhere on the residual subset $\left\{e^{i t}: t \in E\right\}$ of the lower half of $C$; on the upper half of $C$, the function is analytic and has modulus 1 . Let

$$
f_{n}= \begin{cases}g+W_{n} & \text { if } w_{n} \text { is finite } \\ 1 / g & \text { if } w_{n} \text { is infinite }\end{cases}
$$


Reverting to the method by which MacLane and Ryan solved their problem in [12], we describe the function $f$ in terms of its Riemann surface. We begin with the simply connected, unbranched sheet $s$ consisting of (i) one copy of the disk $\Delta$; (ii) one copy $s_{n}$ of the disk $\left\{\left|w-w_{n}\right|<1\right\}$, corresponding to each positive integer $n$; in case $w_{n}=\infty$, we replace this disk with the annulus $\{1<|w|<2\}$, slit along the negative real axis for the sake of simple connectivity; (iii) narrow ribbons $r_{n}$, each connecting the disk in (i) to the domain $s_{n}$ in (ii).

For each index $n$, the Riemann surface $S_{n}$ for the function $f_{n}$ overlies either the disk $s_{n}$ in (ii) or the domain $|w|>1$, depending on whether $w_{n}$ is finite or infinite. In each of the surfaces $S_{n}$ we make one short radial boundary slit ending at the image (under the mapping $f_{n}$ ) of some point $e^{i \theta}$ where $f_{n}$ is analytic; without loss of generality we may assume that the endpoint of the slit does not lie over the juncture of the disk (or annulus) $s_{n}$ with the ribbon $r_{n}$. We make a corresponding slit in $s_{n}$, and we attach the surface $S_{n}$ to $s$ by a crossconnection along the slit.

If $\tilde{f}$ is a conformal mapping of $\Delta$ onto the Riemann surface $s \cup\left(U S_{n}\right)$ thus obtained, and if $P$ denotes projection onto $\mathbf{C}$, then the composite function $f=$ $P \circ \tilde{f}$ has the desired properties.

\section{References}

[1] Arakeljan, N.U.: Uniform and tangential approximation by analytic functions. - Izv. Akad. Nauk Armjan. SSR Ser. Mat. 3, 1968, 4-5, 273-286. (Russian, Armenian, and English summaries).

[2] Cargo, G.T.: Some topological analogues of the F. and M. Riesz uniqueness theorem. J. London Math. Soc. (2) 12, 1976, 67-74.

[3] Collingwood, E.F., and A.J. Lohwater: The theory of cluster sets. - Cambridge Tracts in Mathematics and Mathematical Physics 56, Cambridge University Press, Cambridge, 1966.

[4] Colwell, P.: Blaschke products. Bounded analytic functions. - University of Michigan Press, Ann Arbor, Michigan, 1985.

[5] HausdorfF, F.: Set theory (translation of the third edition of 1937). - Chelsea Publishing Company, New York, 1957.

[6] HeINs, M.: Selected topics in the classical theory of functions of a complex variable. Athena Series: Selected Topics in Mathematics. Holt, Rinehart and Winston, New York, 1962.

[7] Herzog, F., and G. Piranian: Sets of radial continuity of analytic functions. - Pacific J. Math. 4, 1954, 533-538.

[8] Kellogg, O.D.: Harmonic functions and Green's integral. - Trans. Amer. Math. Soc. 13, 1912, 109-132.

[9] Kuratowski, K.: Topology. Vol. I (translated). - Academic Press, New York-London; Państwowe Wydawnictwo Naukowe, Warsaw, 1966.

[10] Lebesgue, H.: Sur les correspondances entre les points de deux espaces. - Fund. Math. 2, 1921, 256-285.

[11] Lusin, N.: Sur la classification de M. Baire. - C. R. Acad. Sci. Paris 164, 1917, 91-94. 
[12] MacLane, G.R., and F.B. Ryan: On the radial limits of Blaschke products. - Pacific J. Math. 12, 1962, 993-998.

[13] Mazurkiewicz, S., and W. Sierpiński: Sur un problème concernant les fonctions continues. - Fund. Math. 6, 1924, 161-169.

[14] Peller, V.V., and S.V. Khrushchëv: Hankel operators, best approximations, and stationary Gaussian processes. - Russ. Math. Surv. 37:1, 1982, 61-144. Translation from Uspehi Mat. Nauk 37, 1982, no. 1 (223), 53-124 (Russian) (1982).

[15] PRIWALOW, I.I.: Randeigenschaften analytischer Funktionen. - VEB Deutscher Verlag der Wissenschaften, Berlin, 1956.

[16] RIEsz, F., and M. RIEsz: Über die Randwerte einer analytischen Funktion. - Quatrième congrès des mathématiciens scandinaves (Stockholm, 1916), 27-44.

[17] Royden, H.L.: Real analysis, Second edition. - Macmillan, New York-London, 1968.

[18] Rudin, W.: Boundary values of continuous analytic functions. - Proc. Amer. Math. Soc. $7,1956,808-811$.

[19] RYAN, F.B.: The set of asymptotic values of a bounded holomorphic function. - Duke Math. J. 33, 1966, 477-484.

[20] RYAN, F.B.: A characterization of the set of asymptotic values of a function holomorphic in the unit disc. - Duke. Math. J. 33, 1966, 485-493.

[21] Tsuji, M.: Potential theory in modern function theory. - Chelsea Publishing Co., New York, 1975.

Wayne State University

Department of Mathematics

Detroit, MI 48202

U.S.A.

Received 15 August 1988
Wayne State University

Department of Mathematics

Detroit, MI 48202

U.S.A.
The University of Michigan Department of Mathematics Ann Arbor, MI 48109-1003 U.S.A. 